of muscle, and the predominant pathogen was $\mathrm{Cl}$. welchii. They provided convincing evidence that penicillin can arrest the progressive myositis, but that, nevertheless, radical surgery is still the main factor in treating the disease.

\section{Penetrating Wound of Abdomen}

It was not felt that there was any special indication for penicillin in penetrating abdominal wounds, for it seemed to us that the deaths were due to physiological reasons rather than to sepsis. Infection of the abdominal wall is an indication of an infective element, and is also a bad prognostic sign as regards late deaths (14 to 28 days). A few such cases were treated by a short systemic course with decided improvement.

\section{Head Wounds}

The percentage of scalp and brain wounds that heal by primary union has always been a high one when operation is performed at special neurosurgical units. In Italy, with greater infectivity of the terrain, this standard tended to deteriorate whenever penicillin was in short supply, but was maintained when penicillin-sulphathiazole powder $(5,000$ units per gramme) was insufflated to surface wounds and into depths of brain. In brain abscesses tubes and instillations formed the method of choice.

\section{Maxillo-facial Wounds ; Eyes ; Burns}

Wounds of the face have hitherto been allowed to heal spontaneously, and at a later date the scar was excised. With the use of penicillin-sulphathiazole powder many face wounds have had primary suture. The resulting scars were admirable, and even if they were not the men were enabled to return to duty and to have cosmetic surgery performed at leisure.

Satisfactory results were obtained in cases of corneal ulcers and abrasions, and conjunctivitis, by 2-hourly drops of sodium penicillin, 1,000 units per c.cm. aq. dest. In 50 cases no staphylococcal, streptococcal, pneumococcal, or gonococcal infection remained after 48 hours of instillations. Infected eye-sockets were treated by local penicillin; panophthalmitis by a systemic course. Blepharitis responded to an ointment of 500 units per gramme of soft lanette wax $(25 \%$ lanette wax in water).

Penicillin-sulphathiazole powder insufflated on to the burned area and covered with tulle gras proved a most excellent dressing for burns. It was not employed frequently, because it used up too much powder.

This was the basis of a report submitted to the D.G.A.M.S. by the Penicillin Control Unit, Central Mediterranean Force. We wish to thank the Director-General for permission to publish this report. We are also grateful to Major-Gen. L. T. Poole (Director of Pathology), Major-Gen. D. C. Monro (Consultant Surgeon), Brigadier Hugh Cairns (Consultant in Neurosurgery), and Sir Howard Florey (Honorary Adviser in Pathology to the Army) for advice and help in our investigation; and to the administrative and medical officers of the R.A.M.C. for their full co-operation.

\section{EARLY DIAGNOSIS OF PERIPHERAL NERVE INJURIES IN BATTLE CASUALTIES BY}

W. RITCHIE RUSSELL, M.D., F.R.C.P.Ed. and Lond. Brigadier; Consultant in Neurology to M.E.F.

\section{AND}

\section{A. B. HARRINGTON, M.B.Aberd. \\ Captain, R.A.M.C.; Graded Physician}

It is the experience of all Nerve Injury Centres that cases are admitted in which there have been delay in diagnosis and errors in early treatment that have gravely prejudiced the subsequent usefulness of the limbs affected. One of us (A. B. H.), for example, has been responsible for the records of cases admitted to a Nerve Injury Centre in the Middle East; the interval after wounding before an associated nerve injury was recognized is given in the accompanying Table. From these figures it is obvious that the methods commonly used in investigating limb injuries soon after wounding often fail to indicate paralysis of one or more of the principal nerves to limbs.
Interval after Injury at which the Presence of a Nerve Lesion was recognized

\begin{tabular}{|c|c|c|c|c|}
\hline \multirow{2}{*}{ Nerve } & \multicolumn{4}{|c|}{ Interval in Weeks after Injury } \\
\hline & $\begin{array}{l}\text { Under } 1 \\
\text { Week }\end{array}$ & $\begin{array}{l}1 \text { to } 4 \\
\text { Weeks }\end{array}$ & $\begin{array}{l}\text { Over } 4 \\
\text { Weeks }\end{array}$ & Totals \\
\hline $\begin{array}{lcccc}\text { Radial } & \ldots & \text { Arm } & & \\
\text { Median } & . & \ldots & . & \ldots \\
\text { Ulnar } & . & \ldots & . & \ldots \\
\text { Two or more nerves } & . & \ldots \\
\text { Other lesions } & \ldots & . . & \ldots \\
\end{array}$ & $\begin{array}{r}11 \\
15 \\
29 \\
15 \\
1\end{array}$ & $\begin{array}{r}8 \\
5 \\
12 \\
2 \\
-\end{array}$ & $\begin{array}{l}4 \\
6 \\
5 \\
1 \\
1\end{array}$ & $\begin{array}{r}23 \\
26 \\
46 \\
18 \\
2\end{array}$ \\
\hline $\begin{array}{l}\text { Sciatic . } \\
\text { Lateral popliteal and branches } \\
\text { Medial popliteal and branches } \\
\text { Other lesions } . .\end{array}$ & $\begin{array}{r}15 \\
12 \\
6 \\
-\end{array}$ & $\begin{array}{l}2 \\
4 \\
3 \\
1\end{array}$ & $\begin{array}{r}1 \\
11 \\
2 \\
-\end{array}$ & $\begin{array}{r}18 \\
27 \\
11 \\
1\end{array}$ \\
\hline Totals & 104 & 37 & 31 & 172 \\
\hline
\end{tabular}

The importance of stressing the need for paying special attention to the early treatment of nerve injuries is indicated by the observation that of 132 cases of nerve injury in the upper limb 61 showed a varying degree of contracture or stiffness of the joints of the fingers. In most of these this disability was due to the effects of nerve injury only and not to associated injury of muscles in the forearm. As will be mentioned later, the maintenance of full range of movement of joints and tendons is the first consideration in the treatment of cases of nerve injury in the upper limb.

The methods of examining cases of nerve injury have been fully described in the M.R.C. War Memorandum No. 7, 1942 - Aids to the Investigation of Peripheral Nerve Injuries"; but surgeons who have to treat battle casualties in forward areas may find useful a description of a few selected tests which they can apply quickly to cases of limb injury while the patient is lying on a stretcher. The most common trick movements are also given.

\section{Rapid Methods of Examination Suitable for Use in Forward Battle Areas}

In most instances the tests needed to demonstrate the integrity of the principal nerves of a wounded limb take less than 60 seconds to apply, and the expert can do the essential tests in less than 30 seconds. When there is nerve injury, however, the tests to locate the lesion may take a further few minutes. If the injuries have caused severe loss of consciousness or profound shock it may be necessary to postpone the examination.

Muscle Tests.-The examination of voluntary action of the muscles is the most accurate method of quick diagnosis, as a few of the muscles supplied by each nerve can be tested in a very short time.. The patient is asked to carry out specific movements against resistance or to maintain posture against resistance (which need be only slight), while, where possible, the muscles contracting or their tendons are observed and palpated to confirm beyond all doubt that the muscles are contracting. The latter part of the test prevents deception by trick movements. Wasting of denervated muscles is a useful guide in chronic cases only. Testing the electrical reactions is of no value in early cases, and is seldom necessary for diagnosis in chronic cases.

Sensory Tests.-The examination of sensation provides valuable confirmatory evidence of the muscle tests, but takes more time, is difficult to carry out in a severely wounded patient, and as a rough preliminary test is less reliable than the motor examination. It is obvious that only the simplest sensory tests can be used in severely injured patients. In this connexion the following principles are worthy of note:

(a) In the majority of cases testing of sensation in the peripheral portion of the limb, in the hand or foot, is the essential part of the examination.

(b) The patient can often himself accurately describe and map out any area of anaesthesia.

(c) Sensory tests should be directed first towards demonstrating the integrity of the main nerves. For example, in the upper limb attention to the palmar aspect of the index and little fingers will test the median and ulnar nerves, while in the lower limb sensation over the dorsum and sole of the foot depends on the lateral and medial popliteal nerves (or their branches) respectively.

(d) Testing with the point of a pin is usually the most useful method, but the stimulus must be vigorous when testing over the thick skin of the palm and sole. Testing for light touch is often best done by stroking the skin lightly with the finger, as a wisp of cotton-wool is useless on thickened skin. 
Sweating Functions.-In peripheral nerve injuries there is anidrosis of the anaesthetic skin areas. When there is general sweating due to heat or shock the dryness of these areas is very obvious and provides a useful objective clinical sign.

Reflex Changes.-Reflex changes provide valuable confirmatory evidence in some cases; for example, there is loss of the biceps-jerk and supinator-jerk in injuries of the upper brachial plexus, loss of the knee-jerk in interruption of the femoral nerve, and loss of the ankle-jerk in medial popliteal nerve palsy.

\section{Wounds of the Upper Limb and Shoulder Girdle}

Radial Nerve.-In wounds of the upper arm causing radial paralysis complete wrist-drop is very obvious provided that movement at the wrist is free. The ability of the patient to maintain the mid-position of the wrist and extension at the metacarpo-phalangeal joints against gravity or resistance (Figs. 1 and 2) excludes gross damage to the radial nerve in the upper arm or to the posterior cord of the brachial plexus. Paralytic wrist-drop is distinguished from flexion contracture or spasm by the resistance to passive dorsiflexion in the latter condition (Fig. 3).

In wounds of the forearm in its proximal half it becomes more necessary to test individual muscles supplied by the radial nerve, such as the extensor digitorum and the extensors of the thumb. These are quickly thrown into activity by extension of the metacarpo-phalangeal joints while the interphalangeal joints are flexed to exclude action of small muscles of the hand (Fig. 2). The tendons of the muscles concerned stand out clearly during contraction. The tendons of the extensor carpi ulnaris and abductor pollicis longus can also be felt when these muscles act. It should be noted that in wounds of the distal half of the forearm an apparent drop-wrist cannot possibly be due to nerve injury, as the muscular branches arise high in the forearm.

Location of Radial Nerve Damage.-Where paralysis of more distal muscles supplied by the radial nerve (extensors of thumb, digits, and wrist) is demonstrated, it is desirable, as in all nerve injuries, to attempt to locate the lesion by examining those muscles supplied from the nerve at a more proximal level. It is convenient to study the muscle supply from below upwards. If there is complete wrist-drop the extensor carpi radialis is paralysed, and the next muscle to test is the brachioradialis, which normally stands out clearly on even a slight effort of flexing the elbow against resistance. More proximally the lateral, medial, and long heads of triceps may be tested respectively by palpating these muscles during attempts to extend the forearm. It should, of course, be noted that severe injuries may make it impossible to do more than carry out the first part of the examination-namely, the testing for wrist-drop.

Sensory testing is of relatively little importance in radial lesions, but a small area of analgesia can usually be demonstrated on the dorsum of the hand between the metacarpals of the thumb and forefinger. It should be noted that the dorsal cutaneous nerve of the forearm is the first branch of the radial nerve, from which it arises at an even higher level than the branches to the triceps. Where this nerve is injured, either through very high radial lesions or after it has left the radial nerve, an area of anaesthesia is found over the back of the forearm and dorsum of the hand.

Trick movements in radial paralysis are: (1) extension of the thumb by the abductor pollicis brevis; (2) extension of the interphalangeal joints by lumbricals and interossei; (3) extension of fingers by flexion of wrist when extensor tendons have become shortened by prolonged splinting.

Median Nerve.--In wounds of the upper arm, injury to the median nerve is easily recognized by the resulting paralysis of many of the forearm flexors. The overlapping of the ulnar nerve supply is the only possible cause of error, and can be avoided by testing flexion of the index finger, which is always a pure median movement (Fig. 4). The flexor carpi radialis can also be easily tested by palpating the tendon at the wrist during attempts to flex the wrist (Fig. 5). The sensory disturbances in median paralysis are very definite, and are constantly present on the palmar aspect of the index finger. Loss of sweating over this finger is also evident.
In wounds of the forearm the median nerve is examined primarily by studying the muscles and skin of the hand. The one muscle of the hand which is constantly supplied by the median nerve alone is the abductor pollicis brevis, and the method of testing this should be carefully studied. The most reliable method is to palpate the muscle (Fig. 6) during strong abduction and extension of the thumb against resistance.

The sensory loss, which is most pronounced over the index finger as described above, is especially important in confirming the diagnosis of medial injury in the forearm or wrist.

Trick movements in median paralysis are: (1) unusually good finger flexion by the ulnar nerve supply; (2) abduction of the thumb by the abductor pollicis longus combined with opponens pollicis when the latter is partly under ulnar control; (3) flexion of the wrist by the abductor pollicis longus (radial nerve) when there is complete median and ulnar paralysis. It is then, however, invariably combined with radial deviation.

Ulnar Nerve.-When the ulnar nerve is divided in the upper arm all the muscles supplied by this nerve are paralysed. Of the forearm flexors supplied, the flexor carpi ulnaris is the easiest to test. On attempting to abduct the little finger strongly this muscle contracts to fix the pisiform, and its tendon can be feilt to tighten at the wrist (Fig. 7). Weakness of flexion of the terminal phalanx of the little finger (flexor profundus) and the characteristic ulnar deformity of the little and ring fingers are also useful signs. There are many methods of testing the small muscles. of the hand supplied by the ulnar nerve. The first dorsal interosseous muscle may be accurately tested by palpating the muscle while the index finger is abducted against resistance. In ulnar paralysis the little finger lies slightly abducted and cannot be adducted to press against the ring finger. Fig. 8 illustrates a quick method of testing the first dorsal interosseous and abductor digiti quinti at the same time, while the adductors can be tested as in Fig. 9. For these tests to be accurate the fingers must not be allowed to extend or flex at the metacarpo-phalangeal joints. The sensory disturbances are easily demonstrable, especially over the little finger, where there is loss of sensation on the palmar surface. There is also loss of sweating over the little and ring fingers.

When the ulnar nerve is divided in the forearm the examination of the hand, as with the median nerve, is the first consideration, as has been mentioned above.

The sensory disturbances have already been described, but one fact must be added-namely, that the dorsal cutaneous branch which supplies the back of the little (and ring) finger leaves the ulnar nerve at about the junction of the middle and lower thirds of the forearm. As a consequence of this, injury distal to this level will not affect sensation of the back of the little finger.

Trick movements in ulnar paralysis are: (1) unusually good flexion of the little finger by median nerve supply; (2) abduction of fingers through the action of the extensor digitorum; (3) adduction of fingers through the action of the flexores digitorum.

\section{Wounds of the Shoulder Region, Upper Thorax, and Neck}

Where there is a possibility of injury to the braehial plexus and its shorter branches, such as musculo-cutaneous and axillary nerves, the examination of the supply of the radial, median, and ulnar nerves should be carried out first. Then, if the local conditions permit, the contraction of the biceps, deltoid, spinati, and other shoulder-girdle muscles should be tested. The detailed examination of these plexus injuries is outside the scope of this paper, but it should be noted that the site and extent of plexus injuries can usually be determined by the application of anatomical facts to the clinical findings.

One interesting trick movement at the shoulder is occasionally seen in cases of complete deltoid paralysis, the patient being able to abduct the arm with apparently normal strength and vigour through the action of the supraspinatus and the clavicular portion of the pectoralis major.

\section{Examination of the Upper Limb when Encased in Plaster-of-Paris}

When the injuries have already been treated by the application of plaster-of-Paris to the forearm and wrist examination is restricted, but the integrity of the radial, median, and ulnar 


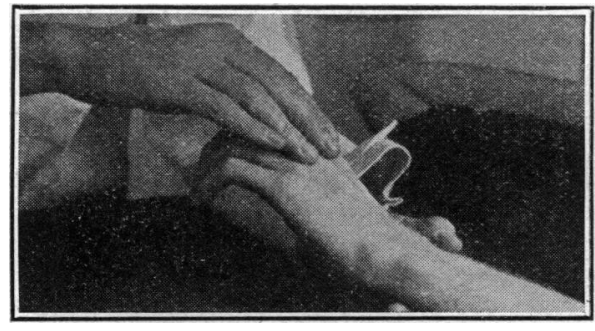

FIG. 1

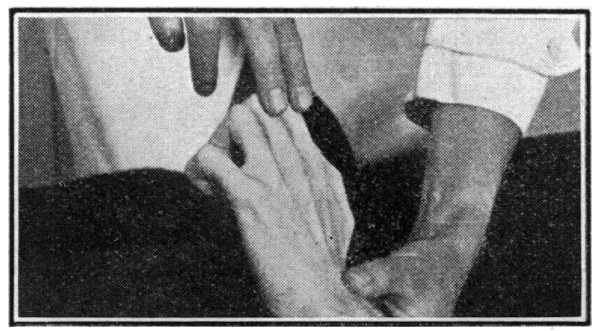

FIg. 2

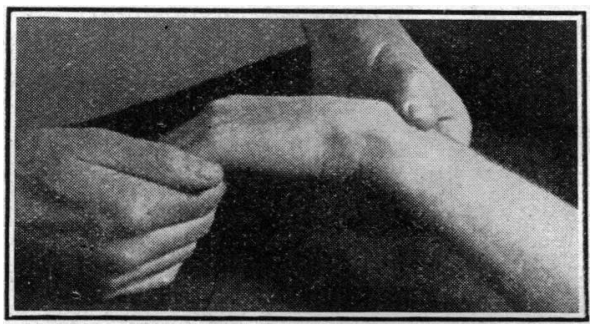

Fig. 3

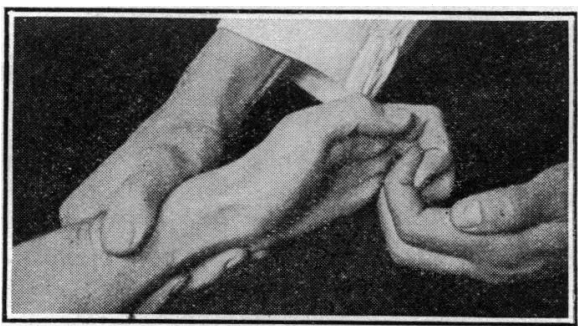

Fig. 4

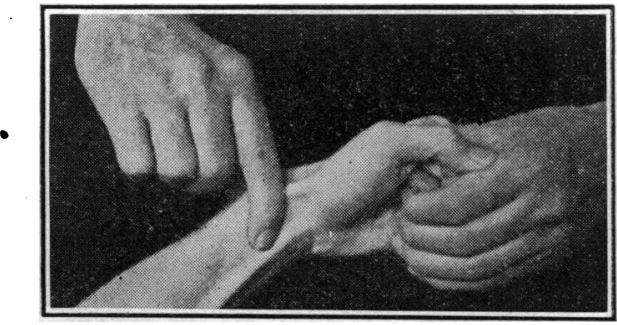

FIG. 5

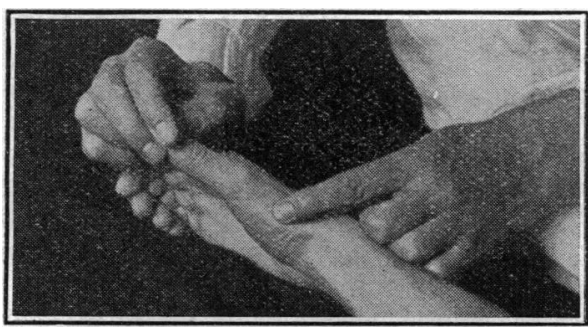

FIG. 6

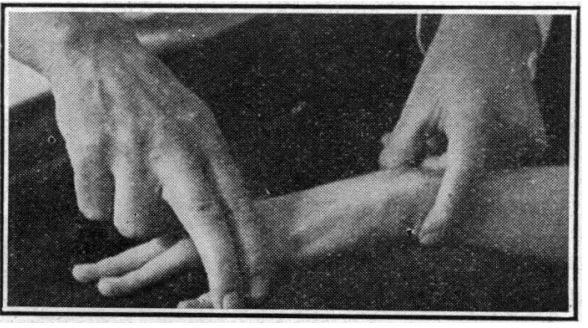

FIG. 7

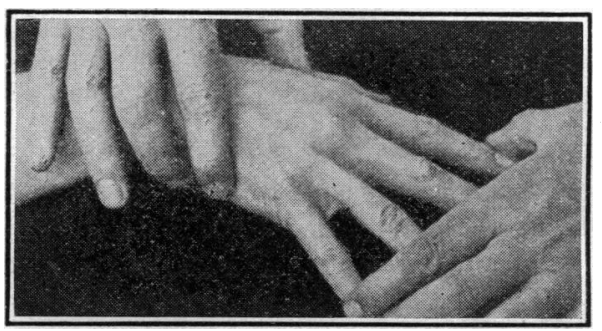

FIG. 8

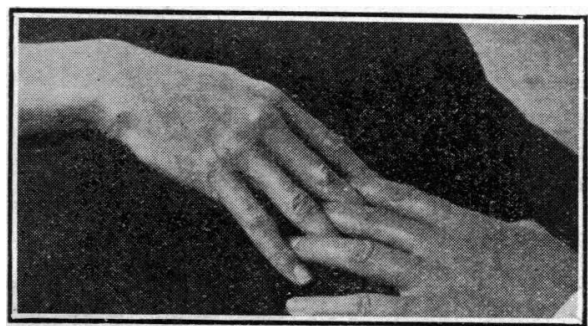

Fig. 9

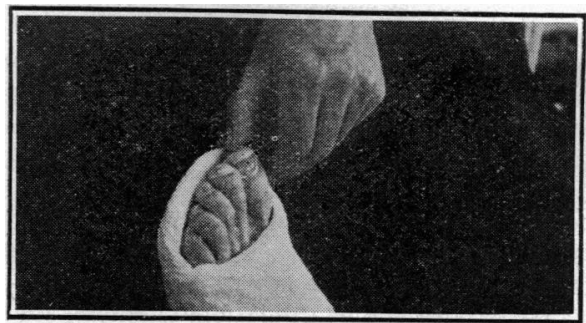

Fig. 10

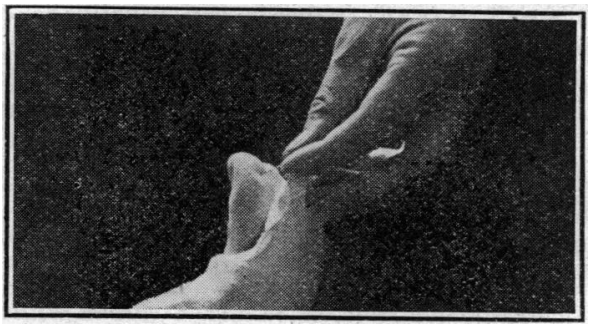

FIG. 11

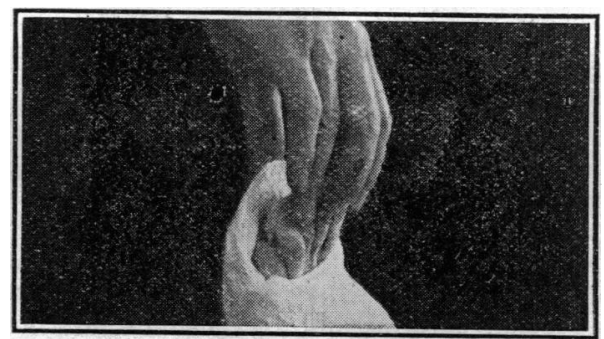

Fig. 12 
nerves can usually be determined in spite of this disadvantage. As will be briefly referred to later, a forearm-and-wrist plaster should extend only to the middle of the palm, to avoid stiffness of the metacarpo-phalangeal joints, and if it has been applied more distally than this it should be trimmed without delay. In such a plaster the extensores digitorum can be readily tested by the method already described (Fig. 2), which eliminates the extending effect of the small muscles of the hand. The upper median nerve can be tested through attempts to flex the index finger, but in the more distal injuries to this nerve sensory testing and anidrosis must be relied on. With regard to the ulnar nerve, flexion of the terminal phalanx of the little finger can be tested, and for the more distal injuries most of the tests described above for the small muscles of the hand can be carried out, as can the sensory tests.

\section{Lower Limb}

The function of the principal nerves to the lower limb can be quickly examined.

Femoral Nerve.-The quadriceps group can be tested either by instructing the patient to "tighten his knee-cap" while the muscles are palpated, or by a slight voluntary effort to raise the heel from the bed; this immediately throws the quadriceps into contraction. In complete femoral paralysis the kneejerk is absent.

Sciatic Nerve.-Conduction in the sciatic nerve is first tested by examining the functions of its two divisions-the medial and lateral popliteal.

Medial Popliteal and its Branches.-The calf muscles are tested by plantar flexion of the foot and toes against resistance. This movement of the toes can be tested even when the foot is encased in plaster (Fig. 10). The sensory loss is over the sole of the foot and plantar aspect of the toes-the latter can also be tested with the foot in plaster (Fig. 11). It should, however, be remembered that the peripheral parts of the toes are supplied by both medial and lateral popliteals, and that, in consequence, lesion of one nerve may give rise to only impaired sensibility in this distribution. The anidrosis of the sole may be a useful sign. It should be noted that wounds of the lower part of the leg and foot which injure the posterior tibial nerve or its terminal cutaneous branches cause anaesthesia, analgesia, and anidrosis of the sole of the foot without paralysis of the long flexors of the toes. Analgesia of the sole of the foot is always a serious disability and, if incomplete, may be associated with causalgic pain. Failure to recognize this disability is therefore a serious though common error.

Lateral Popliteal and its Branches.-Paralysis of the lateral popliteal nerve causes complete drop-foot, which is very obvious owing to the patient's inability to maintain the foot in a position of dorsiflexion after it has been passively moved into this position. This method of testing excludes spasm of the calf muscles as a source of the drop-foot. The sensory loss covers a large part of the dorsum of the foot and lateral aspect of the leg. With the limb encased in plaster, dorsiflexion of the toes provides a useful indication that the nerve is not severed (Fig. 12).

The musculo-cutaneous branch of the lateral popliteal nerve supplies the evertors (peronei) and most of the sensory supply, while the anterior tibial branch supplies the remaining dorsiflexors of foot and toes and a small area of skin between the hallus and the neighbouring toe.

In wounds of the sciatic nerve in the gluteal region and upper thigh the gluteal muscles and the hamstrings require individual testing, but this is rarely practicable on first examination.

\section{Method of Recording Nerve Function}

A record of nerve function soon after injury is of great importance, but unfortunately may take more time than the examination. It is obvious that if nerves are exposed in the wound the position in which they are seen and their condition -whether severed, bruised, or apparently intact-must always be recorded. An apparently intact nerve may temporarily lose all power of conduction. In the recording of clinical tests negative as well as positive observations are highly important.

If time permits, a modified muscle chart greatly adds to the value of the record, especially where there is paralysis. A chart such as the following, for example, would be useful in a case of radial nerve damage in the lower third of the upper arm :

\section{Median nerve \\ Ulnar nerve. \\ Radial nerve-triceps \\ Brachioradialis \\ Ext. carp. rad. \\ Ext. digitorum}

+
+
+
nil
nil
nil

The code, which consists in using the figures 0 to 5 for the strength of muscle contraction, is especially useful ; but it is not so suitable for acute cases, as a recently wounded man cannot be expected to contract his muscles strongly. Disturbances of sensation and sweating should be described briefly. It may be emphasized that even brief records such as the above are of great value in the subsequent treatment of the case, as they will enable. a later recorder to ascertain whether the condition is improving spontaneously or whether it is remaining unchanged, or whether delayed palsies are occurring, due to complications or pressure from plaster, etc.

\section{Early Treatment}

Upper Extremity.-Excessive splinting has done untold harm during the war, especially through its effect in causing stiffness of the joints of the hand. Plasters applied to the forearm should not extend beyond the middle of the palm of the hand, so that the metacarpo-phalangeal joints remain free. In radial palsy the fingers should never be supported by rigid splinting, as this quickly causes irretrievable damage to the finger-joints. The traditional view that paralysed muscles should be kept constantly in a position of relaxation should be discarded, and it is not now practised in Nerve Injury Centres. Light removable splints are used, and at least once daily are taken off so that joints can be passively moved.

In the forward surgery of nerve injuries it is best to make no attempt to support paralysed arm muscles except by using a sling, provided that the patient is warned not to allow the affected muscles to be overstretched. The need for splinting can be decided upon when the case reaches a Nerve Injury Centre. It is, however, highly important that, where possible, the patient be instructed to maintain the range of movement of joints, especially those of the finger, either by active or by passive movements. This prevents the condition of "frozen" hand, which quickly leads to a damaging vicious cycle as follows: Hand kept immobile $\rightarrow$ joints become stiff $\rightarrow$ hand becomes painful $\rightarrow$ patient even more unwilling to move hand, etc. Early movement of joints and active use of the injured 
limb are, when possible, the best treatment for the paralysed muscles, and prevent the development of painful stiffness and various forms of neurotic hesitation in using the limb. In high median lesions passive flexion of the index finger is especially important to avoid stiffness of this finger in extension. In wounds of the brachial plexus the use of a sling and a soft pad in the axilla, if the deltoid is paralysed, combined with regular movements, is all the treatment of the resulting paralysis required during the first two.or three weeks until the case reaches a special centre.

Lower Extremity.-The early treatment of injuries to the nerves of the lower extremity consists simply in maintaining, if possible, the range of movement of the joints. The only support likely to be required is for drop-foot, and, unlike that of the upper extremity, this palsy should be supported as soon as possible. A cradle should be used to relieve the pressure of bed-clothes and to allow movement of the limb. If the sole of the foot is anaesthetic it must be protected from injury, and when there is sufficient recovery to allow walking it is important to provide only well-fitting light boots without irregularities of the sole. The patient should be told of this need to protect anaesthetic skin from both injury and burns.

Finally, as with all traumatic war surgery, it must be stressed that attention to the morale of the patient is most important. As the medical officer has little time to give individual attention to cases of nerve injury, much depends on the massage department. The well-handled man is obviously keen to overcome his disability as much as possible. He moves the joints with vigour and confidence, and maintains the maximum use of his injured limb.

\section{Summary}

Quick neurological methods of examining wounded limbs for nerve injury, suitable for use by. surgeons in a forward battle area, are described and illustrated.

Simple methods of recording are given.

In early treatment the dangers of splinting the fingers are emphasized. Early active or passive movement is valuable. Continual full relaxation of paralysed muscles is harmful.

Our thanks are due to Col. R. A. Broderick, D.S.O., M.C., for permission to refer to cases in No. 27 General Hospital.

\section{ACUTE HAEMOLYTIC ANAEMIA REPORT OF A CASE PRESENTING HITHERTO UNREPORTED FEATURES$$
\text { BY }
$$

\section{J. P. CURRIE, M.D.}

Royat Infirmary, Glasgow

The purpose of this paper is to record a case of acute hacmolytic anaemia occurring in a male adult, showing the rare phenomenon of auto-agglutination* and the possible presence of koth an autohaemolysin and an autohaemolysinogen.

\section{The Literature}

It is outside the scope. of this communication to review the whole of the literature relevant to this rare type of anaemiaa task which has been recently completed by Dameshek and Schwartz. Nevertheless a brief summary of the growth of our knowledge of this syndrome would appear to be a satisfactory point of departure.

Credit for the recognition of haemolytic jaundice as an entity apart from liver or bile-duct disease must be given to Hayem (1898) and Minkowski (1900). No further progress was made until 1907, when Chauffard demonstrated that in cases of chronic

\footnotetext{
* Autohaemolysin:-A haemolysin which is capable of acting against the blood corpuscles of the animal in whose serum it circulates. Auto-agglutinin:-An agglutinin which is capable of agglutinating the blood corpuscles of the animal in whose serum it circulates. Isohaemolysin:-A haemolysin capable of acting against the blood corpuscles of an animal of the same species. Isoagglutinin:-An agglutinin in the blood serum of an individual which is capable of agglutinating the blood corpuscles of another animal of the same species. In humans these include the agglutinins which correspond to the well-known agglutinogens: A, B, and the wh factor. (These definitions are in accordance with Ehrlich's
hh
} lateral-chain theory.) hereditary acholuric icterus with splenomegaly the erythrocytes were abnormally fragile in hypotonic solution. Between 1907 and 1914 several authors-e.g., Widal, Abrami, and Brulédescribed a type of acholuric jaundice without a hereditary factor and occurring either alone or in the course of various diseases. These cases they called "acquired haemolytic icterus." About the same time Chauffard, Troisier, and Vincent (1908) described a third type of case differing from the other two in that an isohaemolysin was present in the serum of their patients during the height of the disease: these cases they called " haemolysinic icterus." Cases of these three types were then subdivided according as to whether they ran an acute, subacute, or chronic course. It is interesting, in view of the case herein reported, to note that in 1912 Roth described a case of haemolytic icterus in which the blood serum contained an auto-agglutinin and an autohaemolysin. He put forward the view that "haemolysinic" was an unjustifiable term, and claimed that the haemolysis was the result of an abnormality of the circulating erythrocytes. Wide controversy now raged, and gradually the existence of an acquired type of haemolytic icterus was gravely doubted.

Interest in the condition was then apparently lost until 1925, when Lederer "discovered" his acute haemolytic anaemia. In spite of the fact that his cases were identical with many previously reported, they were accepted as examples of a "new" disease. His paper made no mention of isohaemolysins or auto-agglutinins indeed, these phenomena seem to have remained forgotten until Davidson's paper in 1932.

It is a matter of no small difficulty to discover how many cases of acute haemolytic anaemia have been reported, the chief difficulty lying in the fact that these cases have been described under many titles-e.g., "atypical anaemia," "atypical pernicious anaemia," "acute pernicious anaemia," and so on. Again, many of the cases described as "acute" have a history which might be regarded as indicative of an exacerbation of a subacute or chronic disease. Dameshek and Schwartz, in their excellent review (1940), list 107 cases which they consider are entitled to the term "acute haemolytic anaemia." I am convinced that, even here, considerable leniency has been shown by the compilers.

The aetiology of many haemolytic anaemias is well knowne.g., bacteria (streptococci), protozoa (malaria), and chemicals (phenylhydrazine). In the majority of cases, however, the cause is unknown; but, broadly speaking, two schools of thought have grown up, thus: (1) that the haemolysis is due to the presence in the serum or tissues of a haemolysin for the patient's own erythrocytes-i.e., an autohaemolysin; (2) that the haemolysis is the result of normal wear and tear, in the circulation, on abnormal (exceedingly fragile) erythrocytes. Present opinion favours Hypothesis 1, and this would appear to be justified by the nature of the marrow reaction alone, since it is unlikely that a marrow which is injured or deficient could respond so well to the demands made of it by the anaemia.

\section{Iso- and Auto-haemolysins}

Isohaemolysins were first demonstrated in a haemolytic state in paroxysmal haemoglobinuria. Here the haemolysin was shown by Donath and Landsteiner (1905) to be activated by cold and to act subsequently at room temperature. Their presence was later demonstrated in several cases of acute haemolytic anaemia by a number of investigators. Dameshek pointed out that although free circulating haemolysins could not be found in every case, that did not rule out their possible presence within the body tissues, and Troisier (1910) and Lieberman and Fenyvessy (1911) offered the explanation that a haemolytic amboceptor became fixed to the erythrocytes, which were thus rendered sensitive to the addition of complement.

While the discovery of isohaemolysins is indicative of the possible or even probable aetiology of the disease, it cannot have such significance as the discovery of autohaemolysins; these, however, have rarely been demonstrable, and then only under abnormal conditions-e.g., at ice-box temperature. The existence of isohaemolysinogens has not been reported.

\section{Auto-agglutinins}

Widal and many other early investigators commented on the concurrence of auto-agglutination in cases of acute acquired 